\title{
Origin of de-swelling and dynamics of dense ionic microgel suspensions
}

\section{Citation}

Romeo, Giovanni, Luna Imperiali, Jin-Woong Kim, Alberto Fernández-Nieves, and David A. Weitz. 2012. "Origin of De-Swelling and Dynamics of Dense lonic Microgel Suspensions." The Journal of Chemical Physics136 (12): 124905. https://doi.org/10.1063/1.3697762.

\section{Permanent link}

http://nrs.harvard.edu/urn-3:HUL.InstRepos:41511283

\section{Terms of Use}

This article was downloaded from Harvard University's DASH repository, and is made available under the terms and conditions applicable to Other Posted Material, as set forth at http:// nrs.harvard.edu/urn-3:HUL.InstRepos:dash.current.terms-of-use\#LAA

\section{Share Your Story}

The Harvard community has made this article openly available.

Please share how this access benefits you. Submit a story.

Accessibility 


\title{
Origin of de-swelling and dynamics of dense ionic microgel suspensions
}

\author{
Giovanni Romeo, ${ }^{1,2, a)}$ Luna Imperiali, ${ }^{2,3}$ Jin-Woong Kim, ${ }^{4}$ Alberto Fernández-Nieves, ${ }^{5}$ \\ and David A. Weitz \\ ${ }^{1}$ Italian Institute of Technology @ CRIB, Napoli, Italy \\ ${ }^{2}$ Department of Materials Engineering and Production, University of Napoli Federico II, Napoli, Italy \\ ${ }^{3}$ Department of Chemical Engineering, K.U. Leuven, Belgium \\ ${ }^{4}$ Department of Applied Chemistry, Hanyang University, 1271, Sal-dong, Sangnok-gu, Ansan-si, \\ Gyeonggi-do 426-791, Korea \\ ${ }^{5}$ School of Physics, Georgia Institute of Technology, Atlanta, Georgia, 30332-0430, USA \\ ${ }^{6}$ Department of Physics \& SEAS, Harvard University, Cambridge, Massachusetts 02138, USA
}

(Received 12 December 2011; accepted 5 March 2012; published online 28 March 2012)

\begin{abstract}
A direct consequence of the finite compressibility of a swollen microgel is that it can shrink and deform in response to an external perturbation. As a result, concentrated suspensions of these particles exhibit relaxation dynamics and rheological properties which can be very different with respect to those of a hard sphere suspension or an emulsion. We study the reduction in size of ionic microgels in response to increasing number of particles to show that particle shrinkage originates primarily from steric compression, and that the effect of ion-induced de-swelling of the polymer network is negligible. With increasing particle concentration, the single particle dynamics switch from those typical of a liquid to those of a super-cooled liquid and finally to those of a glass. However, the transitions occur at volume fractions much higher than those characterizing a hard sphere system. In the supercooled state, the distribution of displacements is non-Gaussian and the dependence of the structural relaxation time on volume fraction is describable by a Volger-Fulcher-Tammann function. () 2012 American Institute of Physics. [http://dx.doi.org/10.1063/1.3697762]
\end{abstract}

\section{INTRODUCTION}

Colloidal suspensions of purely repulsive particles may undergo a liquid to glass transition similarly to molecular systems. For particles characterized by a hard-sphere (HS) interaction, the phase behaviour is dictated only by one parameter: the volume fraction $\phi$. As $\phi$ increases, particle rearrangements slow down and the characteristic time for structural relaxation $\tau$ increases accordingly. At random close packing, $\phi_{r c p}^{h s} \simeq 0.64$, particles reach the maximum packing density and $\tau$ diverges: ${ }^{1}$ the hard sphere system undergoes dynamic arrest. The system also undergoes a glass transition, usually at lower volume fractions. If the inter-particle potential is soft, the glass transition can occur at volume fractions that are higher than random close packing; ${ }^{2}$ the way this transition is approached may significantly differ from the case of hard spheres. ${ }^{3,4}$

Experimentally, one way of designing a system with a soft inter-particle potential is by using microgel particles. Indeed, the intrinsic compressibility of the polymer network of a microgel plays a major role in dictating the phase behavior. These particles are prepared by chemically cross-linking a polymer to form gel particles with sub-micron size. Highly swollen microgels are compressible and can be packed up to very high concentrations, conveniently described by a generalized volume fraction $\zeta=n \cdot v$, where $n$ is the particle number concentration and $v$ is the volume of the particle measured at $n \rightarrow 0$. For these dilute suspensions $\zeta=\phi$. How-

\footnotetext{
a)Electronic mail: giovanni.romeo@ iit.it.
}

ever, $\zeta$ can attain values larger than one, reflecting that microgel particles can shrink; in such a case microgels are not at swelling equilibrium since they have absorbed all the solvent that is available. In this case, $\zeta$ is a measure of the compression of the particles relative to their equilibrium size in dilute conditions.

The size of a fully swollen particle and its softness can be controlled in the polymerization process by adjusting the cross-link density and, in some cases, by tuning external stimuli such as temperature ${ }^{5,6}$ If the polymer that constitutes the particles contains ionic groups, the degree of swelling can also be controlled by $p \mathrm{H}$ and electrolyte concentration. ${ }^{7,8}$ These characteristics make the system highly versatile and tunable.

In the case of ionic microgels, two phenomena can be responsible for particle shrinkage as $\zeta$ increases, namely steric compression and ion-induced de-swelling. Steric compression occurs when $\zeta$ increases above $\phi_{r c p}^{h s}$ and particles reduce in size due to the physical constraints imposed by the neighboring particles. However, in ionic microgels de-swelling may occur even at volume fractions below $\phi_{r c p}^{h s} .{ }^{9,10}$ In fact, the size of a particle depends on the relative concentration of counterions inside and outside the particle. Since the electric charge is distributed within the polymer network, most of the counterions are located inside the microgel, with only a small fraction outside the particle. However, when $\zeta$ increases, the volume available outside the particles decreases, and the relative balance between the inside and outside ion-concentration decreases. This determines a change in the osmotic pressure that can lead to particle de-swelling even before $\phi_{r c p}^{h s}$. From 
this point of view, the effects of both ion-induced de-swelling and steric compression on particle shrinkage need to be quantified in order to evaluate their relative importance in dictating the phase behavior and the dynamics in the dense state.

In this paper we investigate particle de-swelling in a system of ionic microgels. Microgel particles are obtained by copolymerization of poly-N-isopropylacrylamide (pNipa), a neutral polymer, and acrylic acid (AAc) which adds ionic groups to the polymer network. The resulting microgels respond to temperature and $p \mathrm{H}$ variations. We work at $7 \leqslant p \mathrm{H} \leqslant 8$ corresponding to complete dissociation of the ionic groups; the equilibrium state of the microgel particle is thus the fully swollen configuration. Then, by means of confocal microscopy and static light scattering, we measure the particle size as a function of particle concentration. We show that particle shrinking begins when particles are physically touching. In fact, we show theoretically that for this system ion-induced de-swelling becomes significant only for $\zeta$ $\gtrsim 0.8$, well above random close packing. This allows a direct correlation between the particle dynamics in the dense state and the generalized volume fraction $\zeta$. The single particle dynamics are measured by means of particle tracking confocal microscopy and dynamic light scattering. The dynamics in the dense state reveal that particle compression results in a glass transition that is approached smoother than in the hard-sphere case. Indeed, the structural relaxation time $\tau$ increases with $\zeta$ following a Vogel-Fulcher-Tammann (VFT) function, in line with recent results. ${ }^{3}$

\section{MATERIALS AND CHARACTERIZATION METHODS}

\section{A. Particle synthesis and characterization}

The microgel particles are synthesized with a standard precipitation polymerization method. ${ }^{11}$ All reagents have been purchased from Sigma Aldrich. A solution of $385 \mathrm{ml}$ of water with $3.69 \mathrm{~g} \mathrm{~N}$-isopropylacrylamide, $0.029 \mathrm{~g} \mathrm{~N}, \mathrm{~N}^{\prime}-$ methylenebisacrylamide (BIS), and $0.29 \mathrm{~g}$ Acrylic acid is introduced in a $500 \mathrm{ml}$ three-neck flask equipped with a stirrer, nitrogen flow, and water cooling. While stirring, the solution temperature is slowly raised to $68{ }^{\circ} \mathrm{C}$ in an environment saturated with nitrogen. At this point we add a $10 \mathrm{ml}$ solution of water with $0.29 \mathrm{~g}$ of the initiator potassium persulfate (KPS). After about ten minutes from the introduction of the initiator, the solution becomes milky, indicating that colloidal nuclei have formed. The system is left reacting for five hours. At the end of the reaction, the suspension is allowed to cool down to $25{ }^{\circ} \mathrm{C}$. To obtain fluorescent particles, we use rhodamine monomer (methacryloxethyl thiocarbonyl rhodamine B or fluor 570; polysciences), which we add during polymerization after $\approx 15 \mathrm{~min}$ from the introduction of the initiator. Both non-fluorescent and fluorescent suspensions are cleaned by dialyzing against pure water for 15 days and freeze-dried afterwards. The resulting powder is then re-dispersed in water at a polymer concentration $c \simeq 15 \mathrm{wt} \%$. This concentrated solution is agitated at $T \sim 27^{\circ} \mathrm{C}$ for about 10 days to allow homogenization between the phases. Samples at different concentrations are finally obtained by dilution from this batch.

\section{B. Particle characterization: Viscometry and titration measurements}

The viscosity $\eta$ of the microgel suspensions is measured with an Ubbelohde tube immersed in a water bath at a temperature of $(25.00 \pm 0.01){ }^{\circ} \mathrm{C}$. We obtain the relative viscosity $\eta_{r}$ $=\eta / \eta_{o}$, with $\eta_{o}$ the viscosity of water, of dilute suspensions for different polymer concentrations at $p \mathrm{H} 7$ and 8. At each polymer concentration, the $p \mathrm{H}$ is adjusted by slowly adding a solution of $\mathrm{NaOH}$. As shown in Figure 1, the relative viscosities as a function of polymer concentration lie on the same curve for both $p \mathrm{H} 7$ and 8 . This suggests that particle size is constant within this $p \mathrm{H}$ range. In fact, these $p \mathrm{H}$ values are well above the $p K_{a} \simeq 4.25$ of the AAc and the carboxyl groups in the microgel are expected to remain dissociated, keeping the microgel in its fully swollen configuration.

Using the Einstein-Batchelor relation, ${ }^{10} \eta_{r}=1+2.5$ $(k \cdot c)+5.9(k \cdot c)^{2}$, we fit the data at a fixed temperature and obtain the intrinsic volume fraction $k=\zeta / c$. Since the suspension density is essentially equal to that of water, $1 \mathrm{~g} \mathrm{~cm}^{-3}$, the intrinsic volume fraction is $k=\zeta / c=v / m_{p}$, with $m_{p}$ the mass of a particle. From viscometry, we obtain $k=220$ and from dynamic light scattering measurements, we find $v=2.1 \mu \mathrm{m}^{3}$. As a result, $m_{p} \simeq 9.7 \times 10^{-15} \mathrm{~g}$. From this value we can calculate the radius of a completely collapsed particle $R_{0} \simeq\left(\frac{3 m_{p}}{4 \pi}\right)^{1 / 3}=130 \mathrm{~nm}$. Since the radius we measure by DLS on a dilute sample at $43.5^{\circ} \mathrm{C}$ and $p \mathrm{H} 3$ is $R=155 \mathrm{~nm}$, we conclude that even in the de-swollen state pNIPA particles contain a high amount of water. This result suggests that at preparation conditions the polymer volume fraction in a microgel particle is $\varphi_{o} \simeq 0.7$, in agreement with previous reports. ${ }^{12-14}$

To determine the particle charge we perform titration experiments on microgel suspensions and use pure water as reference. $\mathrm{NaOH}$ is slowly added to the microgel suspension and the change in $p \mathrm{H}$ is recorded. The net polymer charge per unit volume $[Q]$ at each $p \mathrm{H}$ value is calculated by

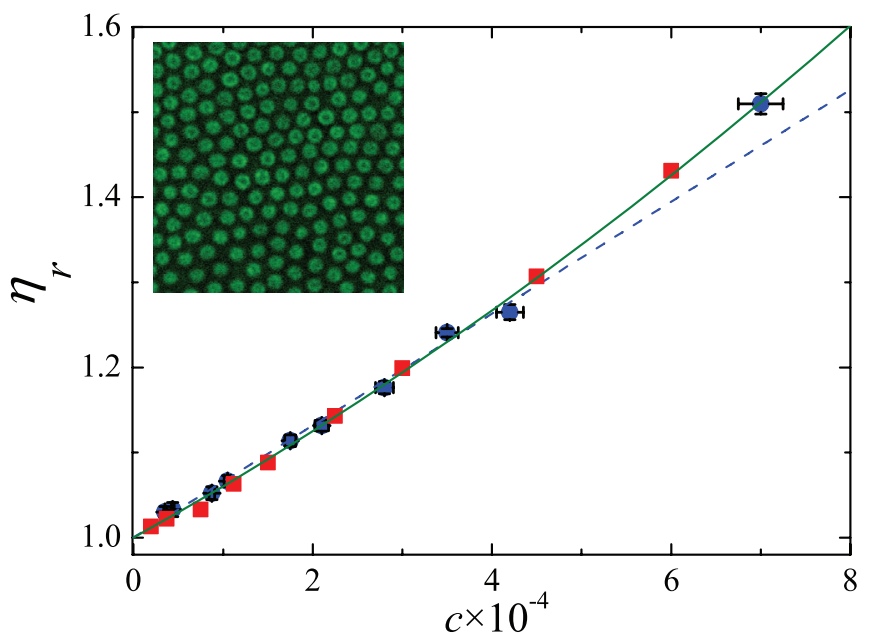

FIG. 1. Dependence of the relative viscosity $\eta_{r}=\eta / \eta_{o}$ on polymer concentration $c$ at $p \mathrm{H} 7$ (circles) and $p \mathrm{H} 8$ (squares). The solid line is a fit to the Einstein-Batchelor relation: $\eta_{r}=1+2.5(k \cdot c)+B(k \cdot c)^{2}$ giving $k=(2.2$ $\pm 0.2) \times 10^{2}$ and $B=4 \pm 2$. Dashed line is a fit to the Einstein equation. The inset shows the fluorescent particles deposited on a glass slide. 


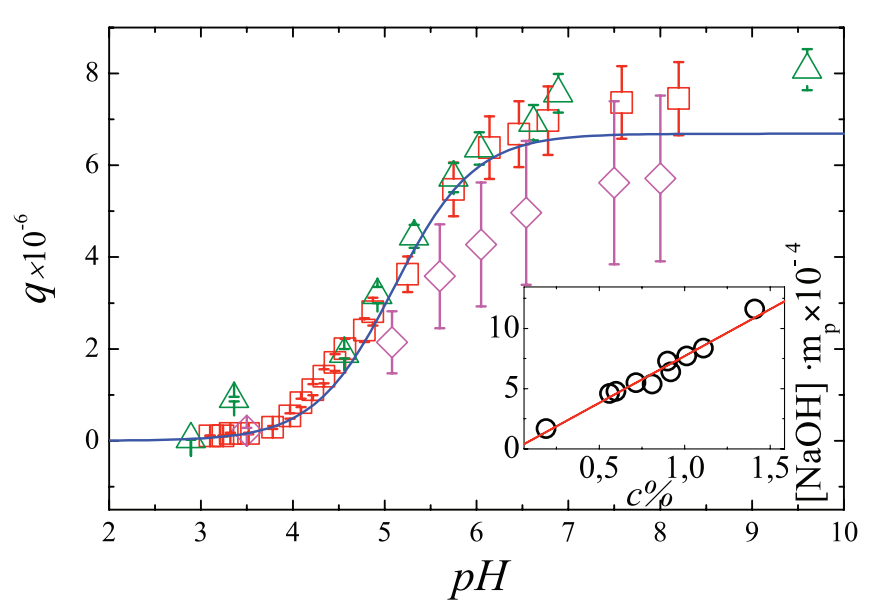

FIG. 2. Number of ionized groups per particle $q$ as function of $p \mathrm{H}$, obtained from titration of three solutions at polymer concentrations $\mathrm{c}=0.19 \%$ (diamonds), $0.56 \%$ (squares), $0.9 \%$ (triangles). The solid line is a fit to the equation $y=\frac{Q}{1+10^{\left(p K_{a}-x\right)}}$ and gives $Q=6.7 \times 10^{6}$ and $p K_{a}=5$. The inset shows the product of the total number of ions per unit volume and particle mass as function of polymer concentration $c$ for samples at $p \mathrm{H} \simeq 7$; the slope of the linear fit is $Q=7.8 \times 10^{6}$

subtracting the moles of $\mathrm{NaOH}$ added to the water and those added to the sample at the same $p \mathrm{H}$ value. The number of dissociated charges per particle $q(p \mathrm{H})$ is then calculated by $q=\frac{[Q]}{n \cdot N_{A}}$, where $N_{A}$ is the Avogadro number and $n=c / m_{p}$ the number of microgels per unit volume in solution. In Figure 2, we show titration curves performed on three samples at different polymer concentrations. Error bars are related to the uncertainty on the determination of $c$. At $p \mathrm{H} \gtrsim 6$, the charge of the particle saturates to a maximum value $Q \simeq 6.7 \times 10^{6}$. This result confirms that no changes in particle size are expected for $p \mathrm{H}$ varying between 7 and 8 , as obtained from viscometry measurements.

In addition, as shown in the inset of Figure 2, the amount of $\mathrm{NaOH}$ required to bring the microgel suspensions to $p \mathrm{H}$ $\simeq 7$ increases linearly with polymer concentration. This suggests that the fraction of free counterions per particle remains unchanged with increasing polymer concentration.

\section{Dynamic light scattering}

We use an ALV/CGS3 Compact Goniometer system operating with a laser of wavelength $\lambda=633 \mathrm{~nm}$ in vacuo and a time correlator ALV/LSE-5003 to perform dynamic light scattering experiments. We measure the normalized time averaged intensity correlation function, $\left\langle g^{(2)}(q, \tau)\right\rangle_{t}$, of the light scattered by the microgel suspension, in homodyne experiments. Here $\tau$ is the lag-time and $q=|\mathbf{q}|=\frac{4 \pi n}{\lambda} \sin \left(\frac{\theta}{2}\right)$ is the modulus of the scattering wavevector, $\mathbf{q}$, with $n=1.33$ the refractive index of water and $\theta$ the scattering angle. From $\left\langle g^{(2)}(q, \tau)\right\rangle_{t}$, the normalized ensemble averaged electric field correlation function $\left\langle g^{(1)}(q, \tau)\right\rangle_{E}$ can be obtained. This measures the normalized dynamic structure factor: $f(q$, $\tau)=F(q, \tau) / S(q)$, where $S(q)=F(q, \tau \rightarrow 0)$ is the static structure factor. Since for ergodic systems, time and ensemble averages are the same, the ensemble averaged field correlation function can be obtained from the time average of the intensity correlation function using the Siegert relation: ${ }^{16}$ $f(q, \tau)=\left\langle g^{(1)}(q, \tau)\right\rangle_{E}=\sqrt{\left\langle g^{(2)}(q, \tau)\right\rangle_{t}-1}$. In dilute solutions, particle correlations are negligible and the dynamic structure factor reduces to $f(q, \tau)=\exp \left(-D_{0} q^{2} t\right)$ with $D_{0}$ the diffusion coefficient of a particle. From this relation, we measure the particle hydrodynamic radius, $R_{h}$, in solutions at polymer concentrations $c \sim 0.01 \mathrm{wt} \%$, which is dilute enough to avoid any particle interactions. At sufficiently small wavevectors, the decay of $f(q, \tau)$ is not influenced by the internal modes of the microgel particle. Indeed, we find that $f(q$, $\tau)$ follows an exponential decay. We fit the relaxation and extract the diffusion coefficient of the particle from the slope of a linear fit of the relaxation frequency, $\gamma=D_{0} q^{2}$, versus $q^{2}$. Using the Stokes-Einstein equation, we obtain $R_{h}=0.8 \mu \mathrm{m}$ for $T=20{ }^{\circ} \mathrm{C}$ and $p \mathrm{H}=7$.

As the particle concentration increases, interactions between particles are no longer negligible and determine particle-particle correlations. These result in short range order which, in reciprocal space, shows up as oscillations in $S(q)$ around 1. For $q \gg q_{\max }$, where $q_{\max }^{-1}$ is related to the characteristic interparticle spacing, $S(q)=1$. If the scattering particles are identical, the dynamic structure factor reduces to

$$
F(\mathbf{q}, \tau)=\frac{1}{N} \sum_{j=1}^{N} \sum_{k=1}^{N}\left\langle\exp \left\{i \mathbf{q} \cdot\left[\mathbf{r}_{j}(0)-\mathbf{r}_{k}(\tau)\right]\right\}\right\rangle_{E},
$$

where $N$ is the number of particles in the scattering volume, and $\mathbf{r}_{j}, \mathbf{r}_{k}$ their positions. The sum in Eq. (1) can be easily separated in two parts, one running over terms $k=j$ which describes single particle motion, $F_{s}(\mathbf{q}, \tau)$, and the other, running over all terms $k \neq j$, describing the correlated motion of different particles, $F_{d}(\mathbf{q}, \tau)$. In the limit $\tau \rightarrow 0, F_{s}(\mathbf{q}, \tau \rightarrow 0)$ $=1$ and $S(\mathbf{q})=1+F_{d}(\mathbf{q}, \tau \rightarrow 0)$; this shows that the departure of $S(q)$ from 1 is a measure of particle correlations, and that $F_{S}(\mathbf{q}, \tau)$ can be obtained at $q$ values such that $S(q) \simeq 1 .^{15}$

For large $q$, when $q \gg q_{\max }, S(q)=1$ and $\left\langle g^{(1)}(q, \tau)\right\rangle_{E}$ directly measures the self dynamic structure factor ${ }^{16}$

$$
\left.f_{s}(\mathbf{q}, \tau)=\frac{1}{N} \sum_{j=1}^{N}\left\langle\exp \left\{i \mathbf{q} \cdot \mathbf{r}_{j}(0)-\mathbf{r}_{j}(\tau)\right]\right\}\right\rangle_{E}
$$

For very high concentrations, particles are confined by their neighbors and are only able to explore an extremely small region of the available space during the experimental time. In such non-ergodic samples $f(q, \tau)$ may be obtained by measuring the intensity of the light scattered by different sub-ensembles of the sample, which assumes that the range of correlation between particles is much smaller than the scattering volume to the one third. In this case, the electric field of the scattered light taken over the full ensemble is a zero mean Gaussian variable, ${ }^{16}$ and the Siegert relation is valid. Experimentally, ensemble averages are performed by changing the position of the vial with respect to the incident beam and then averaging in time. However, a much simpler and less tedious procedure was suggested by Pusey and van Megen ${ }^{17}$ to obtain $f(\tau)$ from $\left\langle g^{2}(\tau)\right\rangle_{t}$

$$
f(\tau)=1+\frac{\langle I\rangle_{t}}{\langle I\rangle_{E}}\left[\sqrt{1+\frac{\left\langle g^{(2)}(\tau)\right\rangle_{t}-\left\langle g^{(2)}(0)\right\rangle_{t}}{\beta}}-1\right],
$$


where $\langle I\rangle_{t}$ is the time averaged intensity obtained during a measurement of $\left\langle g^{(2)}(\tau)\right\rangle_{t}$, for a fixed sample orientation, $\langle I\rangle_{E}$ is the ensemble averaged intensity obtained by rotating the sample, and $\beta=\left\langle g^{(2)}(\tau \rightarrow 0)\right\rangle_{E}-1 \leq 1$ depends on the collection optics. It is straightforward to observe that Eq. (3) reduces to the Siegert relation for ergodic samples where $\langle I\rangle_{E}$ $=\langle I\rangle_{t},\left\langle g^{(2)}(0)\right\rangle_{t}=1+\beta$. For our apparatus, in which the collection optics is a three-mode optical fiber, $\beta=0.4$. Non ergodicity can be easily verified experimentally since the $\tau$ $\rightarrow 0$ intercept of $\left\langle g^{(2)}(\tau)\right\rangle_{t}$ is smaller than that of $\left\langle g^{(2)}(\tau)\right\rangle_{E}$ and $\left\langle g^{(2)}(\tau \rightarrow \infty)\right\rangle_{t}$ does not decay to zero in the experimental time.

To extract the mean square displacement of the particles from a scattering experiment it is necessary to construct a model for the dynamic structure factor. If it is assumed that particle displacements are Gaussian distributed, Eq. (2) reduces to

$$
f_{s}(q, \tau)=\exp \left\{-\left(q^{2} / 6\right) \cdot\left\langle\Delta r^{2}(\tau)\right\rangle\right\}
$$

with

$$
\left\langle\Delta r^{2}(\tau)\right\rangle=\left\langle\left[\mathbf{r}_{j}(\tau)-\mathbf{r}_{j}(0)\right]^{2}\right\rangle
$$

the particle mean square displacement (MSD). For nonGaussian distributions, Eq. (4) represents the second order approximation of a Taylor expansion of Eq. (2). Non-Gaussian behavior can be measured by the magnitude of the next non zero term in the expansion ${ }^{16}$

$$
\begin{aligned}
f_{s}(q, \tau)= & \exp \left\{-\left(q^{2} / 6\right) \cdot\left\langle\Delta r^{2}(\tau)\right\rangle\right. \\
& \left.+\frac{q^{4}}{360}\left[3\left\langle\Delta r^{4}(\tau)\right\rangle-5\left\langle\Delta r^{2}(\tau)\right\rangle^{2}\right]\right\} .
\end{aligned}
$$

The $q^{4}$ coefficient suggests a definition for a nonGaussian parameter: $\alpha_{2}(\tau)=\frac{3\left\langle\Delta r^{4}\right\rangle}{5\left\langle\Delta r^{2}\right\rangle^{2}}-1$.

Usually, non-Gaussian distributions become relevant at the structural relaxation time, ${ }^{18-20}$ while they are negligible for much shorter or longer lag times. In this study the structural relaxation time is observed in the time region investigated by confocal microscopy. We thus neglect non-Gaussian effects in DLS measurements and use Eq. (4) to extract the mean square displacement from the self dynamic structure factor. To verify that this relation holds in concentrated samples we measure $\left\langle g^{(1)}(q, \tau)\right\rangle$ at different $q$ values and calculate the resulting MSD curves from Eq. (4). As shown in Figure 3 for a sample in the concentrated regime, $\zeta \simeq 1.2$, the collapse of the MSD curves calculated at different wavevectors on a single curve confirms that non-Gaussian effects are negligible at lag-times $\tau$ investigated in DLS experiments. The relative electric field correlation functions $\left\langle g^{(1)}(q, \tau)\right\rangle$ from which the MSD are extracted are reported in the inset of Figure 3.

We also find that for $\zeta \gtrsim 1.2$, a peak at low $q$ in the scattered intensity appears suggesting that interparticle correlations are no longer negligible. We identify the position of this peak with $q_{\max }$. In the concentration range $4.8 \gtrsim \zeta$ $\gtrsim 1.3$ the peak moves in the range $8 \gtrsim q_{\max } \gtrsim 5 \mu \mathrm{m}^{-1}$. In this concentration range our experiments are performed at $q \simeq 9 \mu \mathrm{m}^{-1}>q_{\max }$. We assume that this $q$ value is high enough to allow us to measure $f_{s}(q, \tau)$. At the same time, $q$ is assumed small enough to neglect the terms of order $q^{4}$

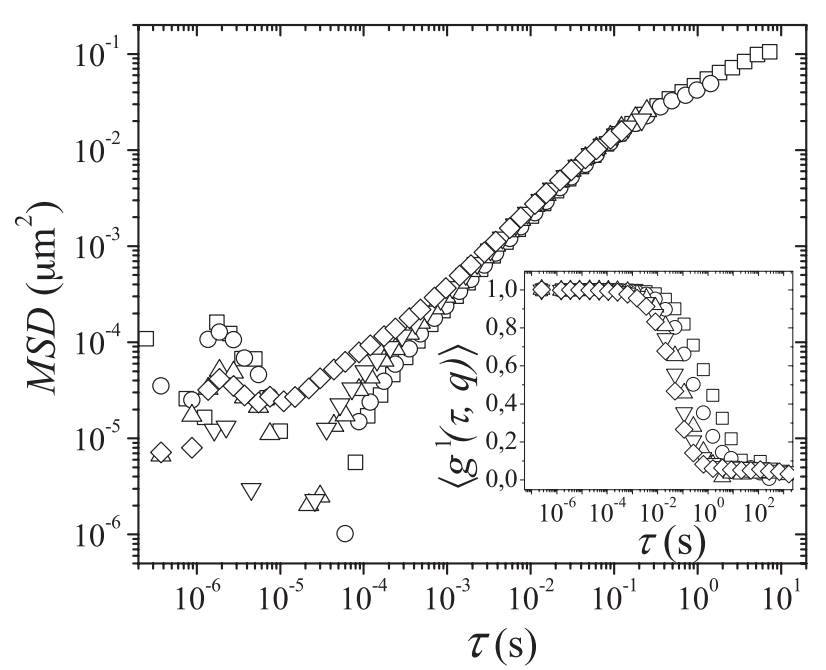

FIG. 3. MSD curves for different wavevectors $q(1 / \mu \mathrm{m}) . q=9$ (squares), 13.2 (circles), 17 (triangles), 20.2 (reverse triangles), 22.9 (diamonds) as a function of lag-time $\tau$ for a sample at $\zeta \simeq 1.2$. In the inset, the time average correlation function of the electric field is reported as function of lag-time. Symbols are the same of the main plot.

and higher in Eq. (6): this is equivalent to the assumption that non-Gaussian effects are minimal in our light scattering experiments.

Finally, since our suspensions are nearly transparent, as we only work with extremely swollen microgels, multiple scattering is negligible.

\section{Static light scattering}

We determine the concentration dependence of the particle radius, $R$, using static light scattering. In general the $q$ dependence of the scattered intensity is a function of both the single particle form factor, $P(q)$, and of the structure factor. For microgels, $P(q)$ depends on $R$, polydispersity, and polymer density distribution within the particle. While the real distribution of polymer density in the particle remains unknown, different scattering experiments ${ }^{13,21-23}$ suggest the presence of a uniformly cross-linked core and a corona in which the polymer density decreases towards the particle periphery. Following Stieger et al. ${ }^{21}$ the particle form factor can be modeled, in reciprocal space, as the product of the form factor of a homogeneous sphere and an error function with a characteristic length scale $\rho$ proportional to the thickness of the corona

$$
\widetilde{P}(q) \propto\left[3 \frac{R q \cos (R q)-\sin (R q)}{(R q)^{3}} \cdot \exp \left(\frac{-\rho^{2} q^{2}}{2}\right)\right]^{2}
$$

This model has been successfully applied to study the structural changes of pNipa-AAc microgels induced by hydrostatic pressure and temperature. ${ }^{24}$ Within the model, the polymer density decreases to half of its core value at $r=R$ and approaches 0 at $r=R+2 \rho$, with $r$ the distance from the particle center. 


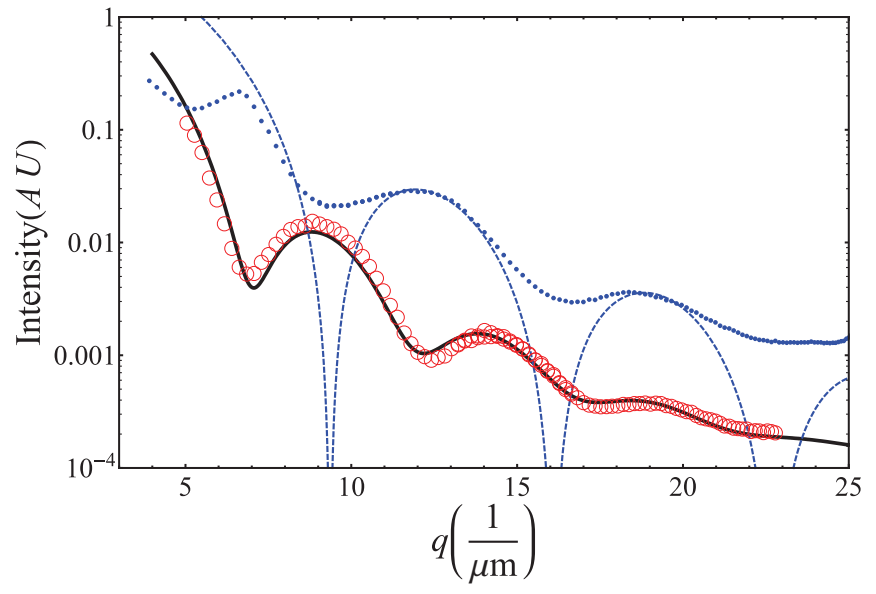

FIG. 4. Scattered intensity (arbitrary units) as function of wavevector $q$ for samples at volume fractions $\zeta=0.15$ (open circles) and $\zeta=3.1$ (solid circles). Solid line is the fitting of data-points of the dilute sample to the form factors of polydisperse inhomogeneous spheres (Eq. (9)) giving a radius $R$ $=0.65 \mu \mathrm{m}$. Dashed line is the form factor of monodisperse inhomogeneous spheres of radius $R=0.48 \mu \mathrm{m}$.

We include polydispersity in the core by assuming a Gaussian distribution for $R$

$$
W(\bar{R}, R, \sigma)=\frac{1}{\sqrt{2 \pi \sigma^{2} \bar{R}^{2}}} \exp \left[-\frac{(R-\bar{R})^{2}}{2 \sigma^{2} \bar{R}^{2}}\right]
$$

where $\bar{R}$ is the mean radius and $\sigma^{2}$ the variance of the distribution. The form factor for dilute suspensions is thus described by

$$
P(q)=\int \widetilde{P}(q) \cdot W(\bar{R}, R, \sigma) d R
$$

As an example, consider the sample at $\zeta=0.15$. The data is well described by Eq. (9), as shown in Figure 4, with $\bar{R}$ $=0.65 \mu \mathrm{m}, \sigma=0.07$ and $\rho=0.045 \mu \mathrm{m}$. From these results we find that the particle radius in dilute conditions is $\bar{R}+2 \rho$ $=0.74 \mu \mathrm{m}$, in good agreement with the hydrodynamic radius, $R_{h}=0.8 \mu \mathrm{m}$, measured by dynamic light scattering.

For concentrated samples, inter-particle correlations are important and $S(q) \neq 1$. As a result, the $q$-dependence of the scattered intensity is hard to model. However, since in our experiments $S(q)$ mainly influences the low $q$ region of the scattered intensity, ${ }^{22}$ we can use the high $q$ region to infer the size of our particles. Indeed, for each particle concentration there are at least two peaks in the form factor within the high $q$ region; thus we can safely estimate the particle radius in dense suspensions by comparing the experimental scattered intensity to the theoretical form factor of monodisperse, inhomogeneous particles. We assume a constant corona thickness $\rho(c)=\rho$ and adjust the concentration-dependent radius, $R(c)$, of the theoretical form factor to match the high $q$ peaks observed experimentally. As an example, the case of $\zeta=3.1$ is shown in Figure 4. Following this procedure we obtain a particle radius $R=0.48 \mu \mathrm{m}$.

\section{E. Confocal measurements of particle radius and dynamics}

We prepare samples at different microgel volume fractions, all containing a small amount, $c \simeq 0.01 \%$, of fluorescent microgels. We use a confocal microscope (ConfoCor 2, Zeiss) working with a water-immersion $63 \times$ objective to directly measure the particle size as a function of particle concentration in dense samples. We acquire 3D stacks, each containing 25 slices, in which successive $2 \mathrm{D}$ images are vertically separated by $0.3 \mu \mathrm{m}$. The acquisition time per frame is about $1 \mathrm{~s}$. To improve visibility and reduce errors due to particles out of focus, we project the 3D stack in a single 2D image. Finally we apply standard image analysis tools ${ }^{25}$ to improve the signal and remove aggregates, and measure the area corresponding to each particle. In Figure 5, a $2 \mathrm{D}$ projection is compared to an image containing the particle contours after processing, for a sample at $\zeta \simeq 1.35$.

Confocal microscopy is also used to measure particle dynamics. We focus on a fixed plane which was always at least $15 \mu \mathrm{m}$ far from the substrate, and capture sequences of images in time. The particle tracking routines for IDL written by Crocker and Grier $^{26}$ are used to determine the locations of the center of mass of the fluorescent particles in each frame, from which particle trajectories are constructed. The mean square displacement (MSD) at time $\tau$ is obtained by fitting the distribution of particle displacements to a gaussian function:

$$
f(\Delta r)=\exp \left(-\frac{\Delta r^{2}}{2 \omega^{2}}\right)
$$

where $f(\Delta r)$ is the probability density function of displacements $\Delta r=|\mathbf{r}(t)-\mathbf{r}(0)|$ and $\omega$ is the standard deviation of the distribution. The MSD is obtained as the standard deviation $\omega$. When particles move freely by Brownian motion, the displacements are random by definition and hence their distribution is perfectly described by a gaussian function: $\omega$ is then mathematically equivalent to the MSD of Eq. (5). When particles movements are constrained, the distribution of displacements is not described by a gaussian. In this case, $\omega$ gives a value of MSD which is representative of the great majority of particle displacements. $^{27}$

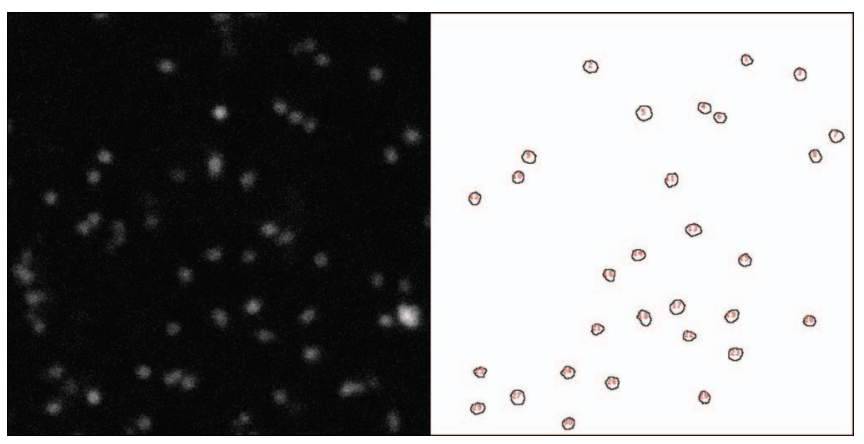

FIG. 5. Left: 2D superposition of 25 slices vertically separated by $0.3 \mu \mathrm{m}$ obtained from a solution of microgels at $c=0.6 \%(\zeta=1.35)$. Right: Contours of the only particles measured by the software after image processing. 


\section{RESULTS AND DISCUSSION}

\section{A. Origin of particle de-swelling}

The equilibrium size of a microgel particle is obtained from the condition of having a zero osmotic pressure difference inside and outside the particle. If the particle surrounding is only water, then ${ }^{28}$

$$
\pi=\pi_{m}+\pi_{e l}+\pi_{i}=0 .
$$

In this equation, the first term, $\pi_{m}$, takes into account the variation in free energy due to polymer/solvent mixing. The second term, $\pi_{e l}$, results from the deformation of the polymer chains inside the microgel with respect to their equilibrium configuration. Both contributions depend on the swelling ratio and can be estimated using Flory's theory for polymer gels ${ }^{28}$

$$
\begin{gathered}
\pi_{m} / k T=-\frac{N_{a}}{v_{s}}\left[\frac{\varphi_{0}}{\alpha}+\ln \left(1-\frac{\varphi_{0}}{\alpha}\right)+\chi\left(\frac{\varphi_{0}}{\alpha}\right)^{2}\right], \\
\pi_{e l} / k T=\frac{N_{c}}{v_{0}}\left[\frac{\varphi_{0}}{2 \alpha}-\frac{1}{\alpha^{1 / 3}}\right],
\end{gathered}
$$

where $k T$ is the thermal energy, $\alpha=\frac{v}{v_{0}}=\frac{\varphi_{0}}{\varphi}$ is the volumetric swelling ratio, $v_{s}=18 \mathrm{~cm}^{3}$ the molar volume of the solvent and $N_{c}$ the effective number of chains in a microgel particle. In addition, $\varphi$ and $v$ are the polymer volume fraction and the particle volume, respectively, and $\varphi_{0}$ and $v_{0}$ are their values in the microgel generation state, the deswollen state in our case. On writing $\pi_{e l}$, it is assumed that the polymer chains are Gaussian. ${ }^{12-14}$

The last term in Eq. (11) describes the additional osmotic pressure arising from the presence of charged groups in the polymer network. In the absence of salt, the main contribution to this ionic term is given by the gas of counterions within the particle. ${ }^{7,8,29}$ Assuming electroneutrality, the number of counterions inside a particle is equal to the total number of ionized groups per particle. As a result

$$
\pi_{i} / k T=\frac{Q}{v_{0} \alpha} .
$$

Using these expressions, we solve Eq. (11) for $T=20^{\circ} \mathrm{C}$ and using the values of $\varphi_{0}, \alpha=\alpha_{\infty}, \chi, v_{0}$, and $Q$ reported in Table I. We find that $N_{c}=2.5 \times 10^{5}$, which is in very good agreement with what we expect from stoichiometry, $N_{c}=3.8 \times 10^{5}$.

Equation (14) assumes that all counterions are trapped inside the microgel particle as a result of the electrostatic attraction of the opposite charges in the polymer network. However, for solutions of polyelectrolyte chains, it is more energetically favorable for the counterions to reside in the bulk solution because of the gain in translational entropy. This effect over- comes the electrostatic interactions with the polyelectrolyte, resulting in an almost absence of counterions within the polyelectrolyte coil. ${ }^{30}$ By denoting the fraction of the counterions outside the coil as $\Gamma$ and introducing the total volume outside the polyelectrolyte, $V_{\text {out }}$, the ionic contribution to the osmotic pressure $\pi_{i}$ can be split in terms of the osmotic pressure inside and outside the polyelectrolyte ${ }^{10,30}$

$$
\pi_{i} / k T=\frac{\pi_{i}^{\text {in }}-\pi_{i}^{\text {out }}}{k T}=\frac{Q}{v_{0} \alpha}(1-\Gamma)-\frac{Q}{V_{\text {out }}} \Gamma .
$$

For cross-linked particles, $\Gamma$ continuously decreases with increasing number of chains $N_{c} .{ }^{30}$ Thus, since the microgel size is generally much larger than that of a polymer chain, the number of chains is huge and most of the counterions are retained inside the particle. Thus, the term $\pi_{i}^{\text {out }}$ is negligible in dilute conditions.

Nevertheless, for sufficiently concentrated suspensions, $V_{\text {out }}$ can be small and $\pi_{i}^{\text {out }}$ can become significant. This can lead to particle de-swelling at volume fractions smaller than random close packing. ${ }^{10,31}$ This effect is clearly seen when expressing $V_{\text {out }}$ through the particle volume fraction, $V_{\text {out }}=\alpha v_{0} \frac{1-\zeta}{\zeta}$, and using the expression for $\pi_{\text {out }}$

$$
\pi_{i}^{\text {out }} / k T=\frac{Q \Gamma}{v_{0} \alpha}\left(\frac{\zeta}{1-\zeta}\right)
$$

This equation predicts that the ionic osmotic pressure outside the particles diverges as $\zeta \rightarrow 1$. However, the importance of this effect is determined by the prefactor $\Gamma$. $\Gamma$ can be calculated by considering that the counterions that leave the particle are those in a peripheral region of thickness $d$, equal to the inverse Debye screening length $k_{i n}^{-1}$. The latter is associated to the decay of the electrostatic Donnan potential at the periphery of the particle: ${ }^{10} \quad k_{i n}^{-1} \simeq\left(4 \pi l_{B} Q / v_{0} \alpha\right)^{-1 / 2}=\sqrt{R^{3} / 3 l_{B} Q}$, with $l_{B}$ $=0.714 \mathrm{~nm}$ the Bjerrum length for water at room temperature. In the approximation that $k_{i n}^{-1} \ll R$, the fraction of counterions that leave the particle is simply given by $\Gamma \simeq \frac{\left(k_{i n}^{-1}+R\right)^{3}}{R^{3}}-1 \simeq 3 k_{i n}^{-1} / R$. For our microgels, $Q \simeq 7$ $\times 10^{6}$ for $p \mathrm{H} \gtrsim 6$ (Figure 2) and $R=0.8 \mu \mathrm{m}$, resulting in $\Gamma=0.022$.

With this value of $\Gamma$, we solve Eq. (11) using Eqs. (12), (13), (15), and (16), together with the values of $N_{c}$ and $v_{0}$ reported in Table I. In this way, we are able to determine the dependence of the swelling ratio $\alpha$ with $\zeta$. In this procedure we assume that $\Gamma$ does not depend on $\zeta$, as suggested by our titration results. We find that $\alpha$ decreases by $10 \%$ of its value at infinite dilution, $\alpha_{\infty}$, when $\zeta \simeq 0.8$. This $10 \%$ is by volume and obviously corresponds to a very small change in size. Note also that the $\zeta$ for which this de-swelling is predicted, is above

TABLE I. Physical and chemical properties of the partially ionic microgel particles: polymer volume fraction in a particle $\varphi_{0}$ at preparation conditions; Flory interaction parameter $\chi$ at $20^{\circ} \mathrm{C}$; particle volume at preparation conditions $v_{0}$; particle volume in the swollen state $v$; swelling ratio at infinite dilution $\alpha_{\infty}$; particle polymer mass $m_{p}$; polymer charge $Q$ per particle; effective number of chains $N_{c}$; counterion screening length $k_{i n}^{-1} ; \Gamma$ is the fraction of counterions that leave the microgel particle at infinite dilution.

\begin{tabular}{cccccccccc}
\hline \hline$\varphi_{0}$ & $\chi$ & $v_{0}\left(\mu \mathrm{m}^{3}\right)$ & $v\left(\mu \mathrm{m}^{3}\right)$ & $\alpha_{\infty}$ & $m_{p}(\mathrm{~g})$ & $Q(\mathrm{e})$ & $N_{c}$ & $k_{i n}^{-1}(\mu \mathrm{m})$ & $\Gamma$ \\
\hline 0.7 & 0.3 & 0.014 & 2.1 & 154 & $9.7 \times 10^{-15}$ & $7 \times 10^{6}$ & $2.5 \times 10^{5}$ & 0.006 & 0.022 \\
\hline \hline
\end{tabular}


random close packing $\phi_{r c p}$, above which other effects associated with particle interpenetration, distortion, and/or compression take place. As a result, these estimations suggest that particle shrinking does not occur before $\phi_{r c p}$ for our microgel particles.

A more rigorous calculation of $\Gamma$ can be performed following the calculations of Denton for the interaction potential between ionic microgels. ${ }^{32}$ The result is an effective interaction, $V$, that depends on $R, Q$, and the Debye screening length $k^{-1}=\sqrt{R^{3} / 3 l_{B} \zeta Q}$. The microgel-microgel interaction thus explicitly depends on $\zeta$ through $k^{-1}$; it softens as $\zeta$ increases. From $V, \Gamma$ can be obtained as ${ }^{32}$

$$
\Gamma=\frac{3}{y}\left(1+\frac{1}{y}\right) e^{-y}\left(\cosh [y]-\frac{\sinh [y]}{y}\right)
$$

with $y=R / k^{-1}$. We observe that for $y \gg 1 \mathrm{Eq}$ (17) reduces to $\Gamma \simeq 3 / 2 y$ in agreement with the approximate solution of Borrega et al. ${ }^{10}$ Using this expression for $\Gamma$ and considering that $R=R_{\infty}\left[\alpha / \alpha_{\infty}\right]^{1 / 3}$, we solve Eqs. (11)-(16) iteratively and we obtain the dependence of $\alpha / \alpha_{\infty}$ on $\zeta$. As shown in Figure 6 , the result agrees very well with our previous estimate based on the model of Borrega et al. ${ }^{10}$

To test the expectations based on these calculations, we measure the microgel radius as a function of $\zeta$, from both confocal images and static light scattering (SLS). Both $R_{\text {conf }}$, the radius extracted from real space confocal images, and $R_{S L S}$, the radius extracted in Fourier space using SLS, show that deswelling begins at $\zeta \approx 1$, as shown in Figure 7; this confirms that counterion-induced de-swelling is negligible for our microgel suspensions when $\zeta$ is smaller than 1. Furthermore, for $\zeta \geq 1$ the available volume is completely filled with microgels and de-swelling proceeds as $R \propto \zeta^{-1 / 3}$ (Figure 7), consistent with what is expected for an isotropic compression. Particle shrinking thus occurs as a result of the steric compression exerted by the neighboring particles.

This can be understood after comparing the relevant length scale for counterion-induced de-swelling, $\kappa_{i n}^{-1}$, and the length of the outer corona of the particles, $\rho$. For our microgels, $\rho \gg k_{i n}^{-1}$. This suggests that before any significant

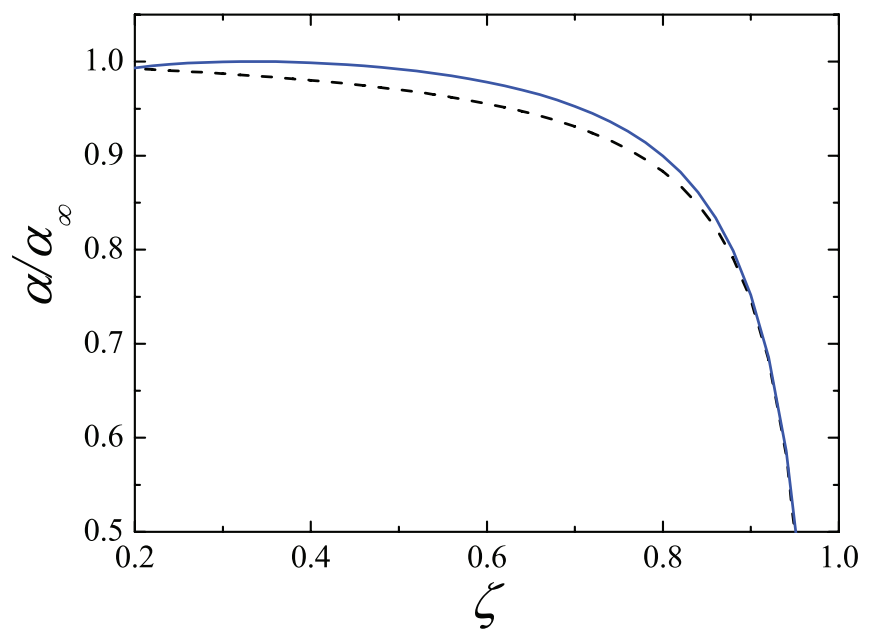

FIG. 6. Prediction of the dependence of the swelling ratio $\alpha$, normalized to its value at infinite dilution $\alpha_{\infty}$, on the generalized volume fraction $\zeta$, as obtained from Eq. (11) (dashed line), and from Eq. (17) (solid line).

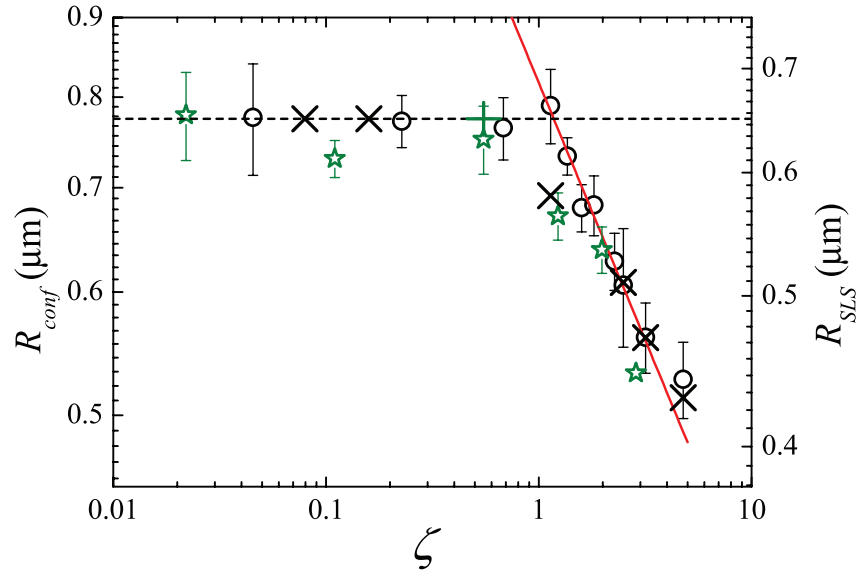

FIG. 7. Dependence of the microgel radius on generalized volume fraction. The radius measured from confocal images $R_{\text {conf }}$ is reported on the left axis for samples at $p \mathrm{H} 7$ (circles) and $p \mathrm{H} 8$ (stars). The radius measured from static light scattering $R_{S L S}$ (crosses) is reported on the right axis for samples at $p H 7$. Vertical axes are scaled to collapse $R_{\text {conf }}$ and $R_{S L S}$ in dilute samples on the same horizontal line (dashed line). Solid line is a plot of the equation $R \propto \zeta^{-1 / 3}$

change in counterion concentration outside the particle happens, the microgel starts shrinking as a result of the steric compression of the corona. We note that the difference in density between particle core and corona also determines a non-uniform distribution of counterions within the microgel particle. In particular, the ions residing outside the particle are less in number with respect to having a microgel with a denser corona. This structure contributes to reduce the dependence of $\pi_{i}^{\text {out }}$ on $V_{\text {out }}$, with respect to what is expected for a homogeneous microgel particle. Indeed, for large $\rho$, the microgel consists of a thick polyelectrolyte outskirt that provides extra volume for the counterions that leave the particle core. As a result, the fraction of counterions in a shell of length $\kappa_{\text {in }}^{-1}$ do not significantly alter $\pi_{\text {out }}$ and counterion-induced de-swelling cannot occur. Our interpretation thus suggests that for counterion-induced de-swelling to be significant, the structure of the microgel should be such that $\kappa_{i n}^{-1} \geq \rho$. Microgels that are more homogeneous than ours would then be required to observe ion-induced de-swelling. Interestingly, this has only been seen with small microgels, for which $\Gamma$ is higher and which are likely to be much more homogeneous than our larger microgels..$^{9,10}$

\section{B. Dynamics in dense suspensions}

The results above establish a firm basis for identifying $\zeta$ with the particle volume fraction, for $\zeta<1$. For larger values of $\zeta, \phi \approx 1$, and the microgels must deswell in order to fit into the available space. Using this direct mapping between $\zeta$ and $\phi$, we explore the dynamics of dense suspensions and observe different mechanisms for particle motion as $\zeta$ increases. For dilute suspensions, $\zeta=0.02$, the slope of the MSD versus time is equal to 1 , as shown in Figure 8(a). The linearity of MSD in time indicates the diffusive character of particle dynamics at these small concentrations. This is also illustrated by the trajectory of one of the particles, which is that of a random walk, as shown in Figure 8(b). Similar results are 


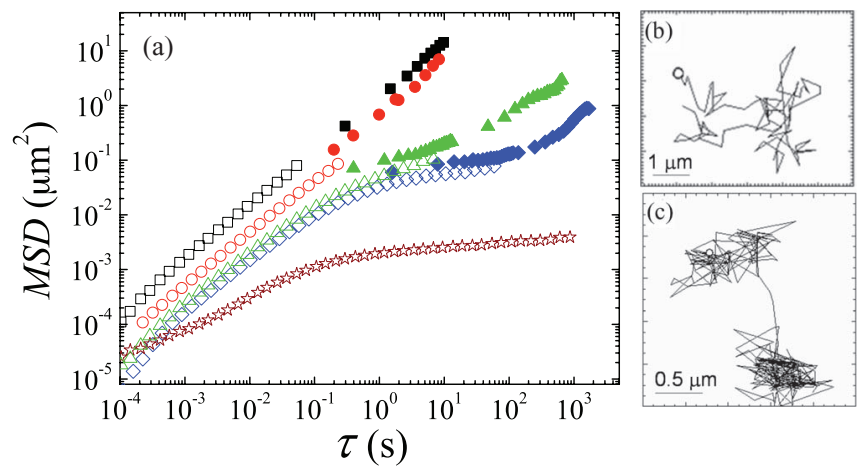

FIG. 8. Left: Dependence of the MSD on lag-delay time $\tau$ for particle volume fractions $\zeta=0.02$ (squares); 0.5 (circles); 1.22 (triangles); 1.96 (diamonds); 2.77 (stars). Open symbols are from DLS and solid symbols from $\mathrm{CM}$. The images on the right represent particle trajectories at $\zeta=0.02$ (top) and $\zeta=1.96$ (bottom) of duration 26 and 500 s respectively.

also obtained at $\zeta=0.5$. However, in this case the diffusion coefficient $D$ is smaller by a factor 3 compared to the case $\zeta=0.02$. Remarkably, for hard spheres at the same volume fractions, this difference in $D$ is a factor of $50 .{ }^{33}$ This result highlights the effect of particle softness on suspension behavior. ${ }^{34}$

For $1<\zeta<2.3$ we observe dynamics typical of supercooled liquids. At short times the motion of the particles is diffusive. However, when the MSD becomes sufficiently large, the motion of the particle is constrained by a cage formed by the neighboring particles. This effect is reflected by an inflection point in the MSD curve, as shown in Figure 8(a). For $\zeta=1.22$, this inflection spans the time range $1 \leq \tau$ $\leq 10 \mathrm{~s}$. For larger times, the MSD of the particles becomes again linear in time. This indicates that, on those time scales, the caging effect does not affect particle motion, as particles are expected to diffuse out of their cages within that time frame. For even larger volume fractions, $\zeta=1.96$, the caging effect is maintained for over two decades in time, as shown in Figure 8(a). Nevertheless, for sufficiently long times, the particles are again able to escape the cage and diffuse away; this is clearly seen in the time evolution of the particle trajectory shown in Figure 8(c). At higher volume fractions, the behavior is sub-diffusive even at the shortest time-scales and cage rearrangement is not observed, as shown in Figure 8(a); in our experimental time window the system is kinetically trapped in a glassy state.

The presence of a cage in hard sphere systems is accompanied by the development of dynamic heterogeneities in the sample: at a fixed lag-time, the particles involved in a cage rearrangement move over longer distances than those still trapped inside their cages. Since particle dynamics are not homogeneous, the distribution of displacements at a fixed lag-time is non-Gaussian. As a result, by quantifying the deviations from gaussianity, we quantify the extent of caging in our samples. This is done by using the parameter $\alpha_{2}(\tau)$, which is zero for a perfectly gaussian distribution and increases as the distribution deviates from Gaussian. For our suspensions, $\alpha_{2}$ remains essentially zero, reflecting Gaussian dynamics (Figure 9(a)), except at $\zeta=1.96$, deep in the supercooled state, where the dynamics are clearly non-Gaussian (Figure 9(b)). At this high $\zeta, \alpha_{2}$ strongly depends on lag-
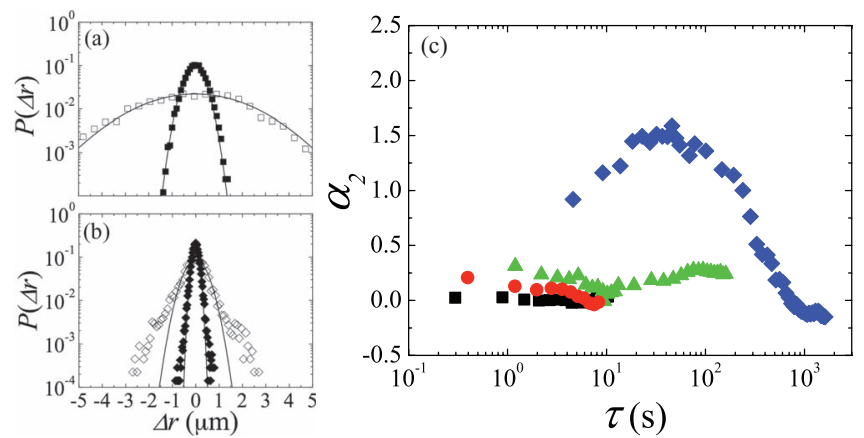

FIG. 9. Left: displacement distributions for samples at $\zeta=0.02$ (top) and $\zeta$ $=1.96$ (bottom). Lines are Gaussian fits to the data. For each sample, solid and empty symbols are for shorter and longer $\tau$ values respectively. Right: Non-Gaussian parameter $\alpha_{2}$ for samples at different $\zeta$ as function of lagtime. Symbols are the same as in Figure 8.

time, as shown in Figure 9(c); it exhibits a maximum at $\tau \simeq 100 \mathrm{~s}$, which is comparable to the structural relaxation time of the sample. This suggests that dynamic heterogeneities, and hence cage rearrangement, are greatest at the structural relaxation of the system, further emphasizing the fundamental role played by these local rearrangements in this relaxation process.

Compared to hard spheres, our results suggest that particle softness strongly broadens the range of volume fractions over which structural relaxation occurs. To quantify this, we define the relaxation time, $\tau_{r}=\tau / \tau_{0}$, as the lag-time value at which a particle displacement is comparable to the particle radius, $\sqrt{M S D} \simeq R$, normalized to the relaxation time of a free diffusing particle $\tau_{0}$. Neglecting the small variations of particle radius with $\zeta$ in the range $2.3>\zeta>1$, for our particles $\tau$ corresponds to the lag-time for a MSD $\simeq 0.4 \mu \mathrm{m}$. We then plot how this time depends on $\zeta$. We find that the $\zeta$-dependence of $\tau_{r}$ follows a modified VogelFulcher-Tammann equation, $\tau_{r}=\exp \left[\frac{A \zeta}{\zeta_{g}-\zeta}\right]$, with $\zeta_{g}=6.87$ the $\zeta$ value corresponding to the glass transition and hence

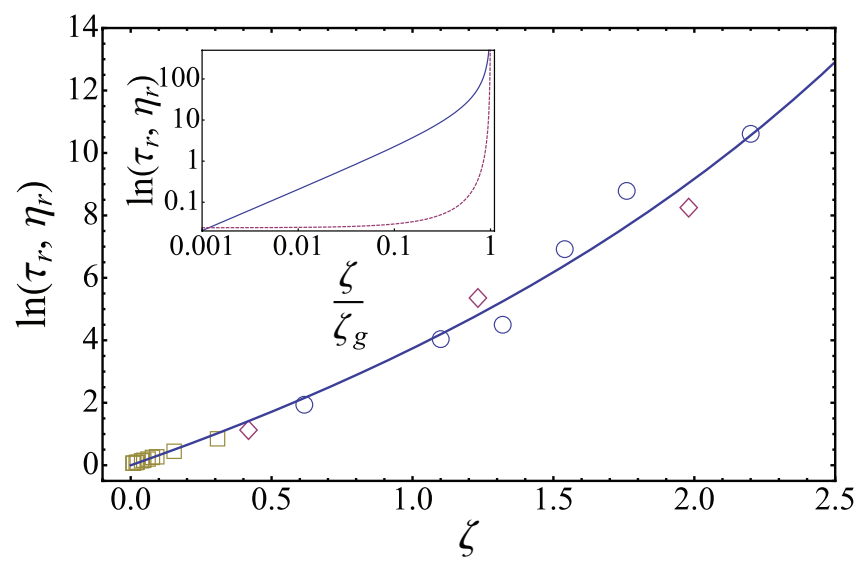

FIG. 10. Structural relaxation time $\tau_{r}$ for microgel solutions at $p \mathrm{H} 7$ (circles) and $p \mathrm{H} 8$ (diamonds) and relative zero-shear viscosity (squares) as function of $\zeta$. Solid line is a fit of the data in the supercooled state $(\zeta>1.1)$ to the VFT equation $\tau_{r}=\exp \left[\frac{A \zeta}{\zeta_{g}-\zeta}\right]$ with $A=22.32$ and $\zeta_{g}=6.87$. The inset shows a $\log -\log$ plot of $\ln \left(\tau_{r}\right)$ as function of the normalized volume fraction $\zeta / \zeta_{g}$. Solid line is the same as in the main figure and the dashed line describes the divergence of hard spheres according to $\tau_{r}=\frac{C}{\left(\phi_{g}-\phi\right)^{2}}$ with $C=0.0098$ and $\phi_{g}=0.64$ (Ref. 35). 
to the divergence of $\tau_{r}$. This result is in line with recent findings for other microgel suspensions. ${ }^{3}$ We note this is very different from hard sphere behavior, with a corresponding $\zeta$-dependence of the structural relaxation time given by $\tau_{r}=\frac{C}{\left(\phi_{g}-\phi\right)^{2}} \cdot{ }^{35}$ To emphasize these differences, we plot our results and the hard sphere expectations in terms of $\zeta / \zeta_{g}$. As shown in the inset of Figure 10, our soft particles display a slower approach to the glass than hard spheres, similar to what is found for strong molecular glass formers. The behavior of our suspensions in the supercooled state, on their approach to the glass, corresponds to an intermediate fragility, characterized by a structural relaxation time that slowly diverges with increasing $\zeta .^{3}$

\section{CONCLUSIONS}

We have shown both experimentally and through calculations based on theoretical models that ionic, swollen pNipa microgel particles do not appreciably shrink with increasing particle concentration as a consequence of counterioninduced de-swelling. This is a consequence of both the small fraction of free counterions $\Gamma$ and of the inhomogeneous structure of the microgels. Indeed our microgel particles shrink because of the steric compression between overlapping chains belonging to neighboring particles. This conclusion has allowed to directly relate the structural relaxation time of the suspension to $\zeta$ and compare it to the hard sphere system. We find that because of particle compressibility, the structural relaxation time and zero-shear viscosity of the microgel suspension increases much slower with particle concentration compared to hard sphere suspensions.

\section{ACKNOWLEDGMENTS}

A.F-N. thanks the Government of Andalucia (Project No. P07-FQM-03116), the FEDER program and the University of Almeria. The work at Harvard was supported by the NSF (Grant No. DMR-1006546) and the Harvard MRSEC (Grant No. DMR-0820484).

${ }^{1}$ G. Brambilla, D. El Masri, M. Pierno, L. Berthier, L. Cipelletti, G. Petekidis, and A. B. Schofield, Phys. Rev. Lett. 102, 085703 (2009).

${ }^{2}$ L. Berthier and T. A. Witten, Europhys. Lett. 86, 10001 (2009).

${ }^{3}$ J. Mattsson, H. M. Wyss, A. Fernández-Nieves, K. Miyazaki, Z. Hu, D. R. Reichman, and D. A. Weitz, Nature (London) 462, 83 (2009).
${ }^{4}$ L. Berthier, E. Flenner, H. Jacquin, and G. Szamel, Phys. Rev. E 81, 31505 (2010).

${ }^{5}$ G. Romeo, A. Fernández-Nieves, H. M. Wyss, D. Acierno, and D. A. Weitz, Adv. Mater. 22, 3441 (2010).

${ }^{6}$ B. Sierra-Martin, Y. Laporte, A. B. South, L. A. Lyon, and A. FernándezNieves, Phys. Rev E 84, 011406 (2011).

${ }^{7}$ A. Fernández-Nieves, A. Fernández-Barbero, B. Vincent, and F. J. de las Nieves, Macromolecules 33, 2114 (2000).

${ }^{8}$ A. Fernández-Nieves, A. Fernández-Barbero, B. Vincent, and F. J. de las Nieves, J. Chem. Phys. 119, 10383 (2003).

${ }^{9}$ B. H. Tan, K. C. Tam, Y. C. Lam, and C. B. Tan, J. Rheol 48, 915 (2004).

${ }^{10}$ R. Borrega, M. Cloitre, I. Betremieux, B. Ernst, and L. Leibler, Europhys. Lett. 47, 729 (1999).

${ }^{11}$ M. J. Snowden, B. Z. Chowdhry, B. Vincent, and G. E. Morris, J. Chem. Soc., Faraday Trans. 92, 5013 (1996).

${ }^{12}$ A. Fernández-Nieves, H. M. Wyss, J. Mattsson, and D. A. Weitz, Microgel Suspensions: Fundamentals and Applications (Wiley-VCH, Weinheim, 2011).

${ }^{13}$ M. Reufer, P. Diaz-Leyva, I. Lynch, and F. Sheffold, Eur. Phys J. E 28, 165 (2009).

${ }^{14}$ A. K. Lele, M. M. Hirve, M. V. Badinger, and R. A. Mashelkar, Macromolecules 30, 157 (1997).

${ }^{15}$ P. N. Pusey, J. Phys. A 11, 119 (1978).

${ }^{16}$ J. K. G. Dhont, An Introduction to Dynamics of Colloids (Elsevier, New York, 1996).

${ }^{17}$ P. N. Pusey and W. van Megen, Physica A 157, 705 (1989).

${ }^{18}$ E. R. Weeks, J. R. Crocker, A. C. Levitt, A. Schofield, and D. A. Weitz, Science 287, 627 (2000).

${ }^{19}$ E. R. Weeks and D. A. Weitz, J. Chem. Phys. 284, 361 (2002).

${ }^{20}$ B. Doliwa and A. Heuer, J. Phys.: Condens. Matter 11, A277-A283 (1999).

${ }^{21}$ M. Stieger, W. Richtering, J. S. Pedersen, and P. Lindner, J. Chem. Phys. 120, 6197 (2004).

${ }^{22}$ M. Stieger, J. S. Pedersen, P. Lindner, and W. Richtering, Langmuir 20, 7283 (2004).

${ }^{23}$ T. G. Mason and M. Y. Lin, Phys. Rev. E 71, 040801 (2005).

${ }^{24}$ J. Lietor-Santos, U. Gasser, R. Vavrin, Z. Hu, and A. Fernández-Nieves, J. Chem. Phys. 133, 034901 (2010).

${ }^{25}$ ImageJ 1.42q (National Institute of Health, USA, 2009).

${ }^{26}$ J. C. Crocker and D. G. Grier, J. Colloid Interface Sci. 179, 298 (1996).

${ }^{27}$ M. T. Valentine, P. D. Kaplan, D. Thota, J. C. Crocker, T. Gisler, R. K. Prud'homme, M. Beck, and D. A. Weitz, Phys. Rev. E 64, 061506 (2001).

${ }^{28}$ P. J. Flory, Principles of Polymer Chemistry (Cornell University, Ithaca, 1953).

${ }^{29}$ A. Fernández-Nieves, A. Fernández-Barbero, and F. J. de las Nieves, J. Chem. Phys. 115, 7644 (2001).

${ }^{30}$ E. Y. Kramarenko and A. R. Khokhlov, Macromolecules 30, 3383 (1996).

${ }^{31}$ B. H. Tan and K. C. Tam, Adv. Colloid Interface Sci. 136, 25 (2008).

${ }^{32}$ A. R. Denton, Phys. Rev. E 67, 011804 (2003).

${ }^{33}$ S. Pyett and W. Richtering, J. Chem. Phys. 122, 34709 (2005).

${ }^{34}$ P. N. Segrè, S. P. Meeker, P. N. Pusey, and W. C. K. Poon, Phys. Rev. Lett. 75, 958 (1995).

${ }^{35}$ D. El Masri, G. Brambilla, M. Pierno, G. Petekidis, A. B. Schofield, L. Berthier, and L. Cipelletti, J. Stat. Mech.: Theory Exp. 2009, 07015 (2009). 